\title{
Einstein-Podolsky-Rosen correlation in gravitational field
}

\author{
Hiroaki Terashima ${ }^{1}$ and Masahito Ueda ${ }^{1,2}$ \\ ${ }^{1}$ Department of Physics, Tokyo Institute of Technology, \\ Tokyo 152-8551, Japan \\ ${ }^{2}$ CREST, Japan Science and Technology Corporation (JST), \\ Saitama 332-0012, Japan
}

\begin{abstract}
For quantum communication in a gravitational field, the properties of the Einstein-Podolsky-Rosen (EPR) correlation are studied within the framework of general relativity. Acceleration and gravity are shown to deteriorate the perfect anti-correlation of an EPR pair of spins in the same direction, and apparently decrease the degree of the violation of Bell's inequality. To maintain the perfect EPR correlation and the maximal violation of Bell's inequality, observers must measure the spins in appropriately chosen different directions. Which directions are appropriate depends on the velocity of the particles, the curvature of the spacetime, and the positions of the observers. Near the event horizon of a black hole, the appropriate directions depend so sensitively on the positions of the observers that even a very small uncertainty in the identification of the observers' positions leads to a fatal error in quantum communication, unless the observers fall into the black hole together with the particles.
\end{abstract}

PACS : 03.65.Ud, 04.20.-q, 03.67.-a, 02.40.-k

Keywords : quantum communication, general relativity, EPR correlation, Bell's inequality

\section{Introduction}

Entanglement is a strange feature of quantum theory and gives rise to a nonlocal correlation called the Einstein-Podolsky-Rosen (EPR) correlation [1, 2] . The EPR correlation was originally discussed to address the foundation of quantum theory. However, it is now widely recognized as a vital resource in quantum communication such as quantum teleportation 3 and quantum cryptography [4, 5]. Thus, to perform precise quantum communication, we 
must understand the properties of the EPR correlation in various physical situations. Recently, a number of articles [6, 7, 8, 9, 10, 11, 12, have discussed how entanglement is affected by the Lorentz transformation in the regime of special relativity [13. For example, the present authors previously discussed the EPR correlation with moving observers who share a common rest frame [8]. In that study, we showed that the perfect anti-correlation of an EPR pair of spins in the same direction deteriorates in the observers' rest frame, and that the degree of the violation of Bell's inequality [14, 15] decreases apparently as the velocity of the observers increases. To utilize the perfect EPR correlation and the maximal violation of Bell's inequality, the moving observers must measure the spins in appropriately chosen different directions; the choice of such directions depends on the velocity of the observers and on that of the particles.

In this paper, we extend these considerations to a regime of general relativity by introducing a gravitational field. In general relativity, a gravitational field is represented by a curved spacetime, which entails a breakdown of the global rotational symmetry. A spin is, on the other hand, known to represent the rotational symmetry of a system. Thus, the spin in general relativity can be defined only locally by invoking the local rotational symmetry of the local inertial frame. In the present paper, we show explicitly how to extract the non-local correlation from the locally defined spins. We also investigate how to extract the non-local correlation beyond the event horizon, which arises in strong gravitational fields and uniquely features the problem of general relativity.

As a consequence of the local definition, a particle moving in curved spacetime is accompanied by a precession of its spin due to the acceleration of the particle by an external force and the difference between local inertial frames at different points. These effects of the particle's motion result in a continuous succession of local Lorentz transformations. Since a Lorentz transformation rotates the spin of the particle according to the Wigner rotation [13, the motion of the particle leads to a continuous succession of local Wigner rotations of the spin, producing spin precession. Spin precession caused by acceleration may be viewed as a generalized Thomas precession in curved spacetime. On the other hand, spin precession caused by a change in the local inertial frame is an effect of spacetime curvature.

Applying both forms of spin precession to a relativistic EPR state near the Schwarzschild black hole, we show that acceleration and gravity deteriorate the perfect anti-correlation in directions that would be the same as 
each other if the spacetime were flat, and that they apparently decrease the degree of the violation of Bell's inequality, as in the case of moving observers in special relativity [8]. To exploit the perfect EPR correlation and the maximal violation of Bell's inequality, the observers must measure the spins in appropriately chosen different directions. Identification of the appropriate directions depends on the velocity of the particles, the curvature of the spacetime, and the positions of the observers. Surprisingly, while the parallel transport in general relativity can define which directions are the same as each other in curved spacetime [16, the appropriate directions are not the same as each other even in this sense. We also show that, near the event horizon of the black hole, the appropriate directions depend quite sensitively on the positions of the observers, because the spin precession induced by the particle's motion becomes very rapid there. Therefore, even a very small uncertainty in the identification of the observers' positions leads to a fatal error in quantum communication near the event horizon. In particular, static observers cannot extract the EPR correlation from circularly moving particles unless they can find their own positions with infinite accuracy. To exploit the EPR correlation on (and beyond) the event horizon, the observers must fall into the black hole together with the particles.

An interesting distinction between the present general-relativistic problem and the special-relativistic one [6, 7, 8, 10, 12, is that in the former the Lorentz transformation arises from the motion of the particle, not from that of the observer, because neither the general coordinate system nor the local inertial frame is changed at each point. This means that we could extend previous work on special-relativistic quantum information to the regime of general relativity only by considering moving particles in a gravitational field. Recently, quantum communications with accelerated observers have been discussed [17, 18] using the Davies-Unruh effect [19] in Minkowski spacetime. However, their protocols have no special-relativistic analogy, since they do not involve the Lorentz transformation. Actually, their resources are not the entanglement between spins.

This paper is organized as follows. Section 2 formulates a spin-1/2 particle in a curved spacetime and shows a spin precession induced by the motion of the particle. As an example, the Schwarzschild spacetime is considered. Section 3 discusses the EPR correlation and Bell's inequality in the Schwarzschild spacetime, and explains how to extract the EPR correlation on and beyond the event horizon subject to an uncertainty in the identification of observers' positions. Section 4 summarizes our results. 


\section{Spin in Curved Spacetime}

\section{$2.1 \quad$ Local Inertial Frame}

In general relativity, a gravitational field is represented by a curved spacetime with metric $g_{\mu \nu}(x)$. To define a spin in the curved spacetime, we introduce a local inertial frame at each point using a vierbein (or a tetrad) $e_{a}^{\mu}(x)$ and its inverse $e^{a}{ }_{\mu}(x)$ defined by [20]

$$
e_{a}^{\mu}(x) e_{b}^{\nu}(x) g_{\mu \nu}(x)=\eta_{a b}
$$

and

$$
e^{a}{ }_{\mu}(x) e_{a}^{\nu}(x)=\delta_{\mu}{ }^{\nu}, \quad e^{a}{ }_{\mu}(x) e_{b}^{\mu}(x)=\delta_{b}^{a},
$$

where $\eta_{a b}=\operatorname{diag}(-1,1,1,1)$ is the Minkowski metric. Here and henceforth, it is assumed that Latin letters run over the four inertial-coordinate labels $0,1,2,3$, that Greek letters run over the four general-coordinate labels, and that repeated indices are to be summed. The general-coordinate labels are lowered by $g_{\mu \nu}(x)$ and raised by its inverse, $g^{\mu \nu}(x)$, defined by $g^{\mu \rho}(x) g_{\rho \nu}(x)=\delta^{\mu}{ }_{\nu}$. The inertial-coordinate labels are lowered by $\eta_{a b}$ and raised by its inverse, $\eta^{a b}$, defined by $\eta^{a c} \eta_{c b}=\delta^{a}{ }_{b}$. The vierbein represents the coordinate transformation from the general coordinate system $x^{\mu}$ to the local inertial frame $x^{a}$ at each point. Therefore, the vierbein and its inverse transform a tensor in the general coordinate system into one in the local inertial frame, and vice versa. For example, a tensor $V_{\rho}^{\mu \nu}(x)$ in the general coordinate system can be transformed into that in the local inertial frame at $x^{\mu}$ via the relation $V_{c}^{a b}(x)=e^{a}{ }_{\mu}(x) e_{\nu}^{b}(x) e_{c}{ }^{\rho}(x) V_{\rho}^{\mu \nu}(x)$. Clearly, the choice of the local inertial frame is not unique, since the inertial frame remains inertial under the Lorentz transformation. The choice of the vierbein therefore has the same degree of freedom known as the local Lorentz transformation. In fact, definitions (11) and (2) remain unaltered under the local Lorentz transformation, i.e.,

$$
\begin{aligned}
& e_{a}{ }^{\mu}(x) \rightarrow e^{\prime}{ }_{a}^{\mu}(x)=\Lambda_{a}^{b}(x) e_{b}{ }^{\mu}(x), \\
& e^{a}{ }_{\mu}(x) \rightarrow e^{\prime a}{ }_{\mu}(x)=\Lambda_{b}^{a}(x) e^{b}{ }_{\mu}(x),
\end{aligned}
$$

where $\Lambda_{a}^{b}(x)=\eta_{a c} \eta^{b d} \Lambda_{d}^{c}(x)$ and $\Lambda_{c}^{a}{ }_{c}(x) \Lambda_{d}^{b}(x) \eta^{c d}=\eta^{a b}$. Although this transformation $\Lambda_{b}^{a}(x)$ is a Lorentz transformation, it has no connection with the Lorentz transformation that is included as a special case in the general coordinate transformation. 
Using the local Lorentz transformation, we can define a particle with spin $1 / 2$ in curved spacetime. It is well known that a "particle" is not defined uniquely in quantum field theory in curved spacetime 21, since the "time" coordinate to define the positive energy is not unique; this non-uniqueness is an origin of particle creation such as Hawking radiation [22]. However, in the present formulation, our particle is specified by the vierbein $e_{0}{ }^{\mu}(x)$, which relates the local time to a global time. A spin- $1 / 2$ particle in curved spacetime is then defined as a particle whose one-particle states furnish the spin-1/2 representation of the local Lorentz transformation, not of the general coordinate transformation. In fact, the Dirac field in the curved spacetime 21] is spinor under the local Lorentz transformation, while it is scalar under the general coordinate transformation. More specifically, consider a massive spin-1/2 particle moving with four-velocity $u^{\mu}(x)=d x^{\mu} / d \tau$, which is normalized as

$$
u^{\mu}(x) u_{\mu}(x)=-c^{2} .
$$

The four-momentum is given by $p^{\mu}(x)=m u^{\mu}(x)$, with $m$ being the mass of the particle. Accordingly, the four-momentum in the local inertial frame becomes $p^{a}(x)=e^{a}{ }_{\mu}(x) p^{\mu}(x)$ using the vierbein. In the local inertial frame at point $x^{\mu}$, the one-particle state in quantum theory is specified by the thirdcomponent $\sigma(=\uparrow, \downarrow)$ of the spin as $\left|p^{a}(x), \sigma ; x\right\rangle$, as in special relativity. This state indicates not a localized state at $x^{\mu}$ with definite momentum $p^{a}(x)$, but rather an extended state whose momentum is $p^{a}(x)$ if it is viewed in the local inertial frame at $x^{\mu}$. By definition, the state $\left|p^{a}(x), \sigma ; x\right\rangle$ transforms as the spin-1/2 representation under the local Lorentz transformation. Note that, in the case of special relativity, a one-particle state $\left|p^{a}, \sigma\right\rangle$ in the spin-1/2 representation transforms under a Lorentz transformation $\Lambda_{b}^{a}$ as [23, 24]

$$
U(\Lambda)\left|p^{a}, \sigma\right\rangle=\sum_{\sigma^{\prime}} D_{\sigma^{\prime} \sigma}^{(1 / 2)}(W(\Lambda, p))\left|\Lambda p^{a}, \sigma^{\prime}\right\rangle
$$

where $D_{\sigma^{\prime} \sigma}^{(1 / 2)}(W(\Lambda, p))$ is a $2 \times 2$ unitary matrix that rotates the spin of the particle according to the Wigner rotation $W_{b}^{a}(\Lambda, p)$. The explicit form of the Wigner rotation is given by

$$
W_{b}^{a}(\Lambda, p)=\left[L^{-1}(\Lambda p) \Lambda L(p)\right]_{b}^{a}
$$

with a standard Lorentz transformation $L^{a}{ }_{b}(p)$,

$$
L_{0}^{0}(p)=\gamma,
$$




$$
\begin{aligned}
L^{0}{ }_{i}(p) & =L^{i}(p)=p^{i} / m c \\
L^{i}{ }_{k}(p) & =\delta_{i k}+(\gamma-1) p^{i} p^{k} /|\vec{p}|^{2},
\end{aligned}
$$

where $\gamma=\sqrt{|\vec{p}|^{2}+m^{2} c^{2}} / m c$ and $i, k=1,2,3$. Therefore, in the case of the curved spacetime, the one-particle state $\left|p^{a}(x), \sigma ; x\right\rangle$ transforms under a local Lorentz transformation $\Lambda_{b}^{a}(x)$ as

$$
U(\Lambda(x))\left|p^{a}(x), \sigma ; x\right\rangle=\sum_{\sigma^{\prime}} D_{\sigma^{\prime} \sigma}^{(1 / 2)}(W(x))\left|\Lambda p^{a}(x), \sigma^{\prime} ; x\right\rangle,
$$

where $W_{b}^{a}(x) \equiv W_{b}^{a}(\Lambda(x), p(x))$ is the local Wigner rotation.

Instead of the one-particle state $\left|p^{a}(x), \sigma ; x\right\rangle$, we could employ Dirac's four-component spinor, which also represents a spin- $1 / 2$ particle. These two are equivalent as far as Lorentz-transformation properties are concerned. For example, the case of moving observers [8] in special relativity was calculated equivalently using Dirac's spinor [7].

\subsection{Spin Precession}

Since the spin of a particle is defined locally relative to the local inertial frame, we next consider the change of the spin when a particle moves from one point to another in curved spacetime. After an infinitesimal proper time $d \tau$, the particle moves to a new point $x^{\prime \mu}=x^{\mu}+u^{\mu}(x) d \tau$. The four-momentum of the particle then becomes $p^{a}\left(x^{\prime}\right)=p^{a}(x)+\delta p^{a}(x)$ in the local inertial frame at the new point, because of changes in both momentum and local inertial frame:

$$
\delta p^{a}(x)=\delta p^{\mu}(x) e^{a}{ }_{\mu}(x)+p^{\mu}(x) \delta e^{a}{ }_{\mu}(x) .
$$

The change in the momentum is given by

$$
\delta p^{\mu}(x)=u^{\nu}(x) d \tau \nabla_{\nu} p^{\mu}(x)=m a^{\mu}(x) d \tau,
$$

where

$$
a^{\mu}(x)=u^{\nu}(x) \nabla_{\nu} u^{\mu}(x)
$$

is the acceleration due to an external force (excluding the gravity). Since $p^{\mu}(x) p_{\mu}(x)=-m^{2} c^{2}$ and $p^{\mu}(x) a_{\mu}(x)=0$ from Eq. (5), we obtain

$$
\delta p^{\mu}(x)=-\frac{1}{m c^{2}}\left[a^{\mu}(x) p_{\nu}(x)-p^{\mu}(x) a_{\nu}(x)\right] p^{\nu}(x) d \tau .
$$


On the other hand, the change in the local inertial frame is given by

$$
\begin{aligned}
\delta e^{a}{ }_{\mu}(x) & =u^{\nu}(x) d \tau \nabla_{\nu} e^{a}{ }_{\mu}(x) \\
& =-u^{\nu}(x) \omega_{\nu b}^{a}(x) e^{b}{ }_{\mu}(x) d \tau \\
& \equiv \chi_{b}^{a}(x) e^{b}{ }_{\mu}(x) d \tau,
\end{aligned}
$$

where

$$
\omega_{\mu b}^{a}(x)=-e_{b}^{\nu}(x) \nabla_{\mu} e^{a}{ }_{\nu}(x)=e^{a}{ }_{\nu}(x) \nabla_{\mu} e_{b}{ }^{\nu}(x)
$$

is the connection one-form (or a spin connection) 20. The second equality in Eq. (15) results from definition (11) and $\nabla_{\mu} g_{\nu \rho}(x)=\nabla_{\mu} \eta_{a b}=0$, giving $\chi_{a b}(x)=-\chi_{b a}(x)$. Substituting Eqs. (13) and (14) into Eq. (10), we obtain

$$
\delta p^{a}(x)=\lambda^{a}{ }_{b}(x) p^{b}(x) d \tau,
$$

where

$$
\lambda_{b}^{a}(x)=-\frac{1}{m c^{2}}\left[a^{a}(x) p_{b}(x)-p^{a}(x) a_{b}(x)\right]+\chi_{b}^{a}(x) .
$$

This is an infinitesimal local Lorentz transformation, since $\lambda_{a b}(x)=-\lambda_{b a}(x)$. That is, when the particle moves, the momentum in the local inertial frame transforms under the local Lorentz transformation

$$
\Lambda_{b}^{a}(x)=\delta_{b}^{a}+\lambda_{b}^{a}(x) d \tau .
$$

Using the unitary operator corresponding to this local Lorentz transformation, the state $\left|p^{a}(x), \sigma ; x\right\rangle$ is now described as $U(\Lambda(x))\left|p^{a}(x), \sigma ; x^{\prime}\right\rangle$ in the local inertial frame at the new point $x^{\prime \mu}$. From Eq. (9), the spin is then rotated according to the local Wigner rotation $W_{b}^{a}(x)$. For the infinitesimal Lorentz transformation (18), the infinitesimal Wigner rotation becomes

$$
W_{b}^{a}(x)=\delta_{b}^{a}+\vartheta^{a}{ }_{b}(x) d \tau,
$$

where $\vartheta_{0}^{0}(x)=\vartheta^{0}{ }_{i}(x)=\vartheta_{0}^{i}(x)=0$ and

$$
\vartheta_{k}^{i}(x)=\lambda_{k}^{i}(x)+\frac{\lambda_{0}^{i}(x) p_{k}(x)-\lambda_{k 0}(x) p^{i}(x)}{p^{0}(x)+m c} .
$$

Its spin-1/2 representation is

$$
\begin{gathered}
D_{\sigma^{\prime} \sigma}^{(1 / 2)}(W(x))=I+\frac{i}{2}\left[\vartheta_{23}(x) \sigma_{x}+\vartheta_{31}(x) \sigma_{y}\right. \\
\left.+\vartheta_{12}(x) \sigma_{z}\right] d \tau
\end{gathered}
$$


with the Pauli matrices $\left\{\sigma_{x}, \sigma_{y}, \sigma_{z}\right\}$ and the unit matrix $I$. We thus obtain the formula for $\left|p^{a}(x), \sigma ; x\right\rangle=U(\Lambda(x))\left|p^{a}(x), \sigma ; x^{\prime}\right\rangle$ as

$$
\begin{aligned}
& U(\Lambda(x))\left|p^{a}(x), \uparrow ; x^{\prime}\right\rangle \\
& =\left(1+\frac{i}{2} \vartheta_{12}(x) d \tau\right)\left|p^{a}\left(x^{\prime}\right), \uparrow ; x^{\prime}\right\rangle \\
& \quad-\frac{1}{2}\left(\vartheta_{31}(x)-i \vartheta_{23}(x)\right) d \tau\left|p^{a}\left(x^{\prime}\right), \downarrow ; x^{\prime}\right\rangle, \\
& U(\Lambda(x))\left|p^{a}(x), \downarrow ; x^{\prime}\right\rangle \\
& =\frac{1}{2}\left(\vartheta_{31}(x)+i \vartheta_{23}(x)\right) d \tau\left|p^{a}\left(x^{\prime}\right), \uparrow ; x^{\prime}\right\rangle \\
& \quad+\left(1-\frac{i}{2} \vartheta_{12}(x) d \tau\right)\left|p^{a}\left(x^{\prime}\right), \downarrow ; x^{\prime}\right\rangle .
\end{aligned}
$$

We emphasize that the change of the spin $\vartheta_{b}^{a}(x)$ is equal neither to $\chi_{b}^{a}(x)$ nor to $\lambda_{b}^{a}(x)$.

By iterating the infinitesimal transformation, we find a transformation formula for a finite proper time. This becomes a Dyson series as in the time-dependent perturbation theory, since $\lambda^{a}{ }_{b}(x)$ 's at different points do not necessarily commute with each other. Suppose that the particle moves along a path $x^{\mu}(\tau)$ from $x_{i}^{\mu}=x^{\mu}\left(\tau_{i}\right)$ to $x_{f}^{\mu}=x^{\mu}\left(\tau_{f}\right)$. Dividing $h=\tau_{f}-\tau_{i}$ into $N$ parts with $x_{(k)}^{\mu} \equiv x^{\mu}\left(\tau_{i}+(h k / N)\right)$ and applying the infinitesimal Lorentz transformation (18) to each part, we obtain the Lorentz transformation for the momentum in the local inertial frame as

$$
\begin{aligned}
\Lambda_{b}^{a}{ }_{b}\left(x_{f}, x_{i}\right) & =\lim _{N \rightarrow \infty} \prod_{k=0}^{N}\left[\delta^{a}{ }_{b}+\lambda^{a}{ }_{b}\left(x_{(k)}\right) \frac{h}{N}\right] \\
& =T \exp \left[\int_{\tau_{i}}^{\tau_{f}} \lambda^{a}{ }_{b}(x(\tau)) d \tau\right],
\end{aligned}
$$

where $T$ is the time-ordering operator, and the exponential refers not to the exponential of each component but to that of the whole matrix. The corresponding Wigner rotation is then given by

$$
\begin{aligned}
W_{b}^{a}\left(x_{f}, x_{i}\right) & =\lim _{N \rightarrow \infty} \prod_{k=0}^{N}\left[\delta^{a}{ }_{b}+\vartheta^{a}{ }_{b}\left(x_{(k)}\right) \frac{h}{N}\right] \\
& =T \exp \left[\int_{\tau_{i}}^{\tau_{f}} \vartheta^{a}{ }_{b}(x(\tau)) d \tau\right] .
\end{aligned}
$$

This formula can be proven by noting that

$$
W_{b}^{a}\left(\Lambda_{1} \Lambda_{2}, p\right)=\left[W\left(\Lambda_{1}, \Lambda_{2} p\right) W\left(\Lambda_{2}, p\right)\right]_{b}^{a}
$$


from the definition (17), and that

$$
\left[\delta^{a}{ }_{b}+\lambda^{a}{ }_{b}\left(x_{(k)}\right) \frac{h}{N}\right] p^{b}\left(x_{(k)}\right)=p^{a}\left(x_{(k+1)}\right)
$$

from the definition of $\lambda_{b}^{a}(x)$.

\subsection{Schwarzschild Spacetime}

As a unique example in general relativity, we consider the Schwarzschild spacetime [25], which is the unique spherically symmetric solution of Einstein's equation in vacuum. In the spherical coordinate system $(t, r, \theta, \phi)$, the metric of this spacetime is given by

$$
\begin{aligned}
d s^{2}=g_{\mu \nu}(x) d x^{\mu} d x^{\nu}= & -f(r) c^{2} d t^{2}+\frac{1}{f(r)} d r^{2} \\
& +r^{2}\left(d \theta^{2}+\sin ^{2} \theta d \phi^{2}\right)
\end{aligned}
$$

where

$$
f(r)=1-\frac{r_{s}}{r}
$$

and the parameter $r_{s}$ is called the Schwarzschild radius. At this radius $r=r_{s}$, the Schwarzschild spacetime has an event horizon where no displacement $d x^{\mu}=(d t, d r, d \theta, d \phi)$ can be timelike $d s^{2}<0$ because of $f\left(r_{s}\right)=0$. The singularity of the metric at the event horizon means not a physical singularity but a breakdown of the coordinate system $(t, r, \theta, \phi)$. The inside of the event horizon $r<r_{s}$ is the Schwarzschild black hole whose mass is given by $M=$ $c^{2} r_{s} / 2 G$. In the Schwarzschild spacetime, it is convenient to choose vierbein (11) such that

$$
\begin{aligned}
e_{0}^{t}(x)=\frac{1}{c \sqrt{f(r)}}, & e_{1}^{r}(x)=\sqrt{f(r)} \\
e_{2}{ }^{\theta}(x)=\frac{1}{r}, & e_{3}^{\phi}(x)=\frac{1}{r \sin \theta}
\end{aligned}
$$

and all the other components are zero. In the following discussions, only non-zero components will be shown. At each point, the 0-, 1-, 2-, and 3-axes are parallel to the $t_{-}, r_{-}, \theta_{-}$, and $\phi$-directions, respectively. This vierbein 
represents a static local inertial frame at each point, because all the components are independent of $t$ and because the components $e_{i}^{t}(x)(i=1,2,3)$ and $e_{0}{ }^{\alpha}(x)(\alpha=r, \theta, \phi)$ are zero. The inverse of vierbein (2) is then given by

$$
\begin{aligned}
e^{0}{ }_{t}(x)=c \sqrt{f(r)}, & e^{1}{ }_{r}(x) & =\frac{1}{\sqrt{f(r)}}, \\
e^{2}{ }_{\theta}(x)=r, & e^{3}{ }_{\phi}(x) & =r \sin \theta .
\end{aligned}
$$

A straightforward calculation shows that the connection one-form (15) becomes

$$
\begin{aligned}
& \omega_{t 1}^{0}(x)=\omega_{t 0}^{1}(x)=\frac{c r_{s}}{2 r^{2}}, \\
& \omega_{\theta 2}^{1}(x)=-\omega_{\theta 1}^{2}(x)=-\sqrt{f(r)} \\
& \omega_{\phi 3}^{1}(x)=-\omega_{\phi 1}^{3}(x)=-\sqrt{f(r)} \sin \theta \\
& \omega_{\phi 3}^{2}(x)=-\omega_{\phi 2}^{3}(x)=-\cos \theta .
\end{aligned}
$$

In this Schwarzschild spacetime, let us consider a particle in a circular motion with a radius $r\left(>r_{s}\right)$ and constant velocity $r d \phi / d t \equiv v \sqrt{f(r)}$ on the equatorial plane $\theta=\pi / 2$. The four-velocity of this particle is given by

$$
u^{t}(x)=\frac{\cosh \xi}{\sqrt{f(r)}}, \quad u^{\phi}(x)=\frac{c \sinh \xi}{r},
$$

where $\xi$ is a rapidity in the local inertial frame defined by

$$
\frac{v}{c}=\tanh \xi
$$

In order for the particle to move in this way, we must apply an external force against the centrifugal force and the gravity. The acceleration (12) due to this external force then becomes

$$
a^{r}(x)=-\frac{c^{2} \sinh ^{2} \xi}{r}\left[1-\frac{r_{s}}{2 r f(r)} \operatorname{coth}^{2} \xi\right] f(r) .
$$

Now, after an infinitesimal proper time $d \tau$, the particle moves by an angle $\delta \phi=u^{\phi}(x) d \tau$ as depicted in Fig. 1. However, the change in the local inertial 


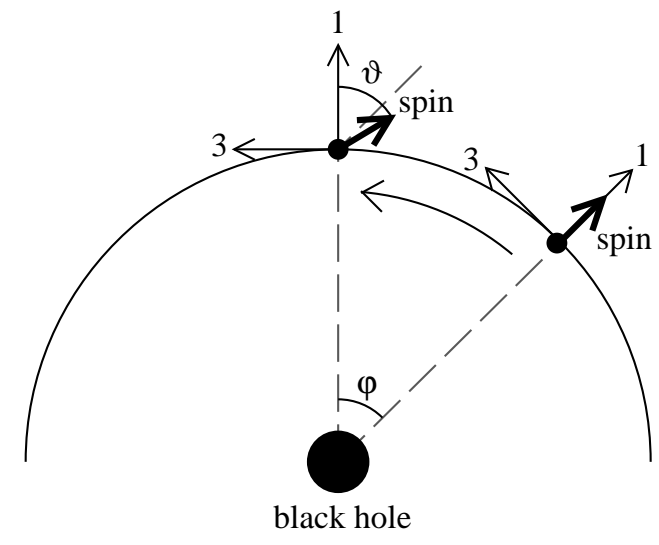

Figure 1: A spin in a circular motion. At each point, the 1- and 3-axes are parallel to the $r$ - and $\phi$-directions, respectively.

frame (14) is not a trivial rotation about the 2-axis,

$$
\varphi^{1}{ }_{3}(x)=-\varphi^{3}{ }_{1}(x)=u^{\phi}(x)=\frac{c \sinh \xi}{r},
$$

since in general relativity a parallel transport that depends on the spacetime curvature is required to compare local inertial frames at different points. The definition (14) shows that the change in the local inertial frame consists of a boost along the 1 -axis and a rotation about the 2 -axis,

$$
\begin{aligned}
& \chi_{1}^{0}(x)=\chi_{0}^{1}(x)=-\frac{c r_{s} \cosh \xi}{2 r^{2} \sqrt{f(r)}}, \\
& \chi_{3}^{1}(x)=-\chi_{1}^{3}(x)=\frac{c \sinh \xi \sqrt{f(r)}}{r} .
\end{aligned}
$$

The infinitesimal Lorentz transformation (17) then becomes

$$
\begin{aligned}
\lambda_{1}^{0}(x) & =\lambda_{0}^{1}(x) \\
& =-\frac{c \cosh \xi \sinh ^{2} \xi}{r}\left[1-\frac{r_{s}}{2 r f(r)}\right] \sqrt{f(r)}, \\
\lambda_{3}^{1}(x) & =-\lambda^{3}{ }_{1}(x) \\
& =\frac{c \cosh ^{2} \xi \sinh \xi}{r}\left[1-\frac{r_{s}}{2 r f(r)}\right] \sqrt{f(r)},
\end{aligned}
$$


which also consists of a boost along the 1-axis and a rotation about the 2-axis. Nevertheless, the momentum in the local inertial frame is constant, $p^{a}(x)=(m c \cosh \xi, 0,0, m c \sinh \xi)$, pointing to the 3 -axis. Finally, the change of the spin defined by Eq. (20) becomes the rotation about the 2-axis through an angle

$$
\vartheta_{3}^{1}(x)=-\vartheta^{3}{ }_{1}(x)=\frac{c \cosh \xi \sinh \xi}{r}\left[1-\frac{r_{s}}{2 r f(r)}\right] \sqrt{f(r)},
$$

as illustrated in Fig. 1, It is important to note that

$$
\vartheta^{a}{ }_{b}(x) \neq \lambda^{a}{ }_{b}(x) \neq \chi_{b}^{a}(x) \neq \varphi^{a}{ }_{b}(x) ;
$$

these three non-equalities result from the boost part of $\lambda^{a}{ }_{b}(x)$, the acceleration of the particle, and the curvature of the spacetime, respectively.

To illustrate this result, we consider a special case, $r_{s}=0$, i.e. Minkowski spacetime. In this case, the change in the local inertial frame is reduced to the rotation about the 2 -axis through an angle

$$
\chi_{3}^{1}(x)=-\chi_{1}^{3}(x)=\frac{c \sinh \xi}{r}
$$

which is nothing but the trivial rotation (39). The change of the spin is also reduced to the rotation about the 2 -axis through an angle

$$
\vartheta_{3}^{1}(x)=-\vartheta^{3}{ }_{1}(x)=\frac{c \cosh \xi \sinh \xi}{r} .
$$

The difference between (46) and (47) gives rise to a precession of the spin called the Thomas precession. When $v / c \ll 1$, the precession angle per $d t=d \tau \cosh \xi$ becomes

$$
\left[\vartheta^{3}{ }_{1}(x)-\chi_{1}^{3}(x)\right] d \tau \sim-\frac{v a}{2 c^{2}} d t
$$

where $a \equiv\left|a^{r}(x)\right|=c^{2} \sinh ^{2} \xi / r$.

\section{$3 \quad$ EPR Correlation and Bell's Inequality}

We now discuss how to exploit the EPR correlation for quantum communication using accelerated particles in a gravitational field. Specifically, we consider a pair of circularly moving particles in the Schwarzschild spacetime, as discussed in the preceding section. 


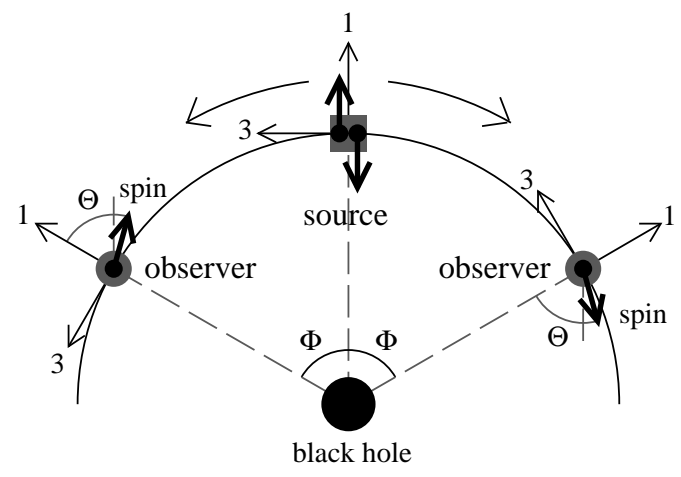

Figure 2: An EPR gedankenexperiment in the Schwarzschild spacetime. Two observers (gray circles) and an EPR source (gray square) are located at $\phi=$ $\pm \Phi$ and 0 , respectively.

\subsection{EPR Correlation}

Consider two observers and an EPR source on the equatorial plane $\theta=\pi / 2$ at a radius $r\left(>r_{s}\right)$ with azimuthal angles $\pm \Phi$ (observers) and 0 (EPR source), as illustrated in Fig. 2. The observers and the EPR source are assumed to be static ("at rest" in the coordinate system $(t, r, \theta, \phi))$ and to use the static local inertial frame (30) to measure or prepare the spin state. Note that the inertial frame is defined at each instant, since the observers and EPR source are accelerated to stay at a constant radius. First, the EPR source emits a pair of entangled particles in opposite directions with constant four-momenta $p_{ \pm}^{a}=(m c \cosh \xi, 0,0, \pm m c \sinh \xi)$ in the spin-singlet state,

$$
\frac{1}{\sqrt{2}}\left[\left|p_{+}^{a}, \uparrow ; 0\right\rangle\left|p_{-}^{a}, \downarrow ; 0\right\rangle-\left|p_{+}^{a}, \downarrow ; 0\right\rangle\left|p_{-}^{a}, \uparrow ; 0\right\rangle\right],
$$

where for notational simplicity we write only the $\phi$-coordinate in the arguments. After a proper time $r \Phi / c \sinh \xi$, each particle reaches the corresponding observer. Using Eq. (44), the Wigner rotation (25) becomes a rotation about the 2-axis

$$
W_{b}^{a}( \pm \Phi, 0)=\left(\begin{array}{cccc}
1 & 0 & 0 & 0 \\
0 & \cos \Theta & 0 & \pm \sin \Theta \\
0 & 0 & 1 & 0 \\
0 & \mp \sin \Theta & 0 & \cos \Theta
\end{array}\right)
$$


where the angle $\Theta$ is given by

$$
\Theta=\Phi \cosh \xi\left[1-\frac{r_{s}}{2 r f(r)}\right] \sqrt{f(r)} .
$$

Note that we do not need the time-ordering operator $T$ in this example, since $\vartheta_{b}^{a}(x)$ is constant during the motion. This Wigner rotation is represented by using the Pauli matrix $\sigma_{y}$ as

$$
D_{\sigma^{\prime} \sigma}^{(1 / 2)}(W( \pm \Phi, 0))=\exp \left(\mp i \frac{\sigma_{y}}{2} \Theta\right) .
$$

Therefore, in the local inertial frames at $\phi=\Phi$ and $-\Phi$, the state is described as

$$
\begin{aligned}
\frac{1}{\sqrt{2}}\left[\operatorname { c o s } \Theta \left(\left|p_{+}^{a}, \uparrow ; \Phi\right\rangle\left|p_{-}^{a}, \downarrow ;-\Phi\right\rangle\right.\right. \\
\left.-\left|p_{+}^{a}, \downarrow ; \Phi\right\rangle\left|p_{-}^{a}, \uparrow ;-\Phi\right\rangle\right) \\
+\sin \Theta\left(\left|p_{+}^{a}, \uparrow ; \Phi\right\rangle\left|p_{-}^{a}, \uparrow ;-\Phi\right\rangle\right. \\
\left.\left.+\left|p_{+}^{a}, \downarrow ; \Phi\right\rangle\left|p_{-}^{a}, \downarrow ;-\Phi\right\rangle\right)\right],
\end{aligned}
$$

as illustrated in Fig. 2. Because the spin-singlet state is mixed with the spin-triplet state, spin measurements in the same direction are not always anti-correlated in the local inertial frames at $\phi= \pm \Phi$ (e.g. in each 1-axis). Clearly, this result includes the trivial rotation of the local inertial frames $\pm \Phi$, as in Eq. (39). To eliminate this spurious effect, we rotate the bases at $\phi= \pm \Phi$ about the 2 -axis through the angles $\mp \Phi$, respectively:

$$
\begin{aligned}
&\left|p_{ \pm}^{a}, \uparrow ; \pm \Phi\right\rangle^{\prime}= \cos \frac{\Phi}{2}\left|p_{ \pm}^{a}, \uparrow ; \pm \Phi\right\rangle \\
& \pm \sin \frac{\Phi}{2}\left|p_{ \pm}^{a}, \downarrow ; \pm \Phi\right\rangle, \\
&\left|p_{ \pm}^{a}, \downarrow ; \pm \Phi\right\rangle^{\prime}=\mp \sin \frac{\Phi}{2}\left|p_{ \pm}^{a}, \uparrow ; \pm \Phi\right\rangle \\
&+\cos \frac{\Phi}{2}\left|p_{ \pm}^{a}, \downarrow ; \pm \Phi\right\rangle .
\end{aligned}
$$

Using these bases, the state is written as

$$
\frac{1}{\sqrt{2}}\left[\operatorname { c o s } \Delta \left(\left|p_{+}^{a}, \uparrow ; \Phi\right\rangle^{\prime}\left|p_{-}^{a}, \downarrow ;-\Phi\right\rangle^{\prime}\right.\right.
$$




$$
\begin{gathered}
\left.-\left|p_{+}^{a}, \downarrow ; \Phi\right\rangle^{\prime}\left|p_{-}^{a}, \uparrow ;-\Phi\right\rangle^{\prime}\right) \\
+\sin \Delta\left(\left|p_{+}^{a}, \uparrow ; \Phi\right\rangle^{\prime}\left|p_{-}^{a}, \uparrow ;-\Phi\right\rangle^{\prime}\right. \\
\left.\left.+\left|p_{+}^{a}, \downarrow ; \Phi\right\rangle^{\prime}\left|p_{-}^{a}, \downarrow ;-\Phi\right\rangle^{\prime}\right)\right],
\end{gathered}
$$

where

$$
\Delta=\Theta-\Phi=\Phi\left\{\cosh \xi\left[1-\frac{r_{s}}{2 r f(r)}\right] \sqrt{f(r)}-1\right\} .
$$

Since the trivial rotation is removed in these bases, we can explicitly see that the relativistic effect deteriorates the perfect anti-correlation in the directions that would be the same as each other if the spacetime were flat. This deterioration is a consequence of the non-equality $\vartheta_{b}^{a}(x) \neq \varphi^{a}{ }_{b}(x)$ in Eq. (45). Of course, our result does not mean a breakdown of the non-local correlation, since the entanglement is invariant under local unitary operations. If we take account of the relativistic effect arising from acceleration and gravity, we can exploit the perfect anti-correlation for quantum communication. More specifically, the observers at $\phi= \pm \Phi$ must rotate the directions of the measurement about the 2-axis through the angles $\mp \Theta$ in their local inertial frames, respectively. It is interesting that the parallel transport in general relativity [16] does not give the directions that maintain the perfect anticorrelation. This occurs because of $\vartheta_{b}^{a}(x) \neq \chi_{b}^{a}(x)$ in Eq. (45).

The value of $\Delta$ as a function of $r_{s} / r$ and $v / c=\tanh \xi$ is shown in Fig. 3. In the non-relativistic limit $v / c \rightarrow 0$ and $r_{s} / r \rightarrow 0$, the angle $\Delta$ becomes

$$
\Delta \sim \Phi\left(\frac{v^{2}}{2 c^{2}}-\frac{r_{s}}{r}\right)
$$

The first term is attributable to acceleration and the second to gravity. Note that they have different signs. Although Eq. (58) holds only for the nonrelativistic limit, we can draw from it the following qualitative physical picture: At the spatial infinity $r \rightarrow \infty$, the gravitational field is so weak that the angle $\Delta$ is positive. However, closer to the event horizon, the gravitational field becomes stronger, thus making $\Delta$ smaller. At a radius $r=r_{0}$ defined by

$$
\left[1-\frac{r_{s}}{2 r_{0} f\left(r_{0}\right)}\right] \sqrt{f\left(r_{0}\right)}=\sqrt{1-\frac{v^{2}}{c^{2}}},
$$

the angle $\Delta$ vanishes, and becomes negative for $r<r_{0}$ (see Fig. 3). In the limit of $v \rightarrow c$, the radius $r_{0}$ becomes $3 r_{s} / 2$; inside this radius, the 


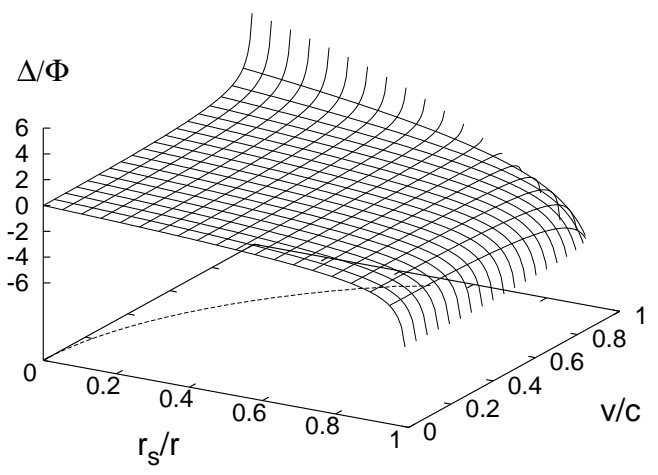

Figure 3: The angle $\Delta$ as a function of $r_{s} / r$ and $v / c=\tanh \xi$. The dotted line at the bottom is the radius $r_{0}$, where $\Delta$ becomes $0 .|\Delta / \Phi|$ is on the order of $10^{-9}$ on the earth if $v \ll 10 \mathrm{~km} / \mathrm{s}$. However, it becomes infinite as $r \rightarrow r_{s}$ or $v \rightarrow c$.

gravitational field is so strong that the acceleration $a^{r}(x)$ for the circular motion must be in the outward direction for any $v$. Right on the event horizon $r \rightarrow r_{s}$, we find $\Delta \rightarrow-\infty$.

\subsection{Uncertainties in Observers' Positions}

We have shown that the observers can, in principle, utilize the EPR correlation by adjusting the directions of measurement. Nevertheless, in practice, such adjustments become difficult near the horizon, since the spin precession (44) is very rapid there. Suppose a classical or quantum uncertainty $\delta \Phi$ exists in the observers' position $\Phi$. The error of the angle $\Theta$ to maintain the perfect EPR correlation then becomes

$$
\delta \Theta=\delta \Phi\left|1+\frac{\Delta}{\Phi}\right|
$$

Quantum communication must tolerate this error by some error-correcting scheme. However, near the horizon $r \sim r_{s}, \delta \Theta$ can be much larger than $\pi$ and thus the observers cannot determine the directions of measurement clearly enough to extract the EPR correlation. Therefore, to utilize the EPR 
correlation, $\delta \Phi$ and $r$ must satisfy at least

$$
\delta \Phi<\pi\left|1+\frac{\Delta}{\Phi}\right|^{-1}
$$

At the horizon $r=r_{s}$, this requirement reduces to $\delta \Phi=0$ because the velocity of the spin precession (44) is infinite. This means that the observers on the horizon could not extract the EPR correlation from the particles, were it not for the infinite accuracy of $\Phi$. More generally, for a given uncertainty $\delta \Phi$, there exists a radius $r_{c}\left(>r_{s}\right)$ such that static observers at $r<r_{c}$ cannot extract the EPR correlation from circularly moving particles.

However, if the observers use different local inertial frames and different particles, they can extract the EPR correlation even at $r<r_{c}$. Note that the divergence of the spin precession (44) comes from the fact that the vierbein (30) and four-velocity (36) become singular at the horizon. Therefore, the observers must choose a vierbein and a four-velocity that avoid the singularities at the horizon. Since these singularities are connected with the breakdown of the coordinate system $(t, r, \theta, \phi)$, we adopt the Kruskal coordinate system [25, in which the metric is not singular at the horizon. The Kruskal coordinates $(T, R)$ are defined from $(t, r)$ by

$$
R^{2}-c^{2} T^{2}=4 r_{s}^{2} \frac{f(r)}{F(r)}, \quad \frac{c T}{R}=\tanh \left(\frac{c t}{2 r_{s}}\right),
$$

where

$$
F(r)=\frac{r_{s}}{r} e^{-r / r_{s}}
$$

In the Kruskal coordinate system $(T, R, \theta, \phi)$, the metric becomes

$$
d s^{2}=-F(r) c^{2} d T^{2}+F(r) d R^{2}+r^{2}\left(d \theta^{2}+\sin ^{2} \theta d \phi^{2}\right),
$$

where the radial coordinate $r$ is now interpreted as a function of $T$ and $R$. In light of this coordinate system, we choose a new vierbein $\tilde{e}_{a}^{\mu}(x)$ as

$$
\begin{array}{rlrl}
\tilde{e}_{0}^{T}(x)=\frac{1}{c \sqrt{F(r)}}, & \tilde{e}_{1}^{R}(x) & =\frac{1}{\sqrt{F(r)}}, \\
\tilde{e}_{2}{ }^{\theta}(x)=\frac{1}{r}, & \tilde{e}_{3}^{\phi}(x)=\frac{1}{r \sin \theta}
\end{array}
$$

which is not singular at the horizon. Using the original coordinate system $(t, r, \theta, \phi)$, we find that this vierbein is related to the static vierbein (30) by 
a local Lorentz transformation: $\tilde{e}_{a}{ }^{\mu}(x)=\tilde{\Lambda}_{a}^{b}(x) e_{b}{ }^{\mu}(x)$, where

$$
\tilde{\Lambda}_{a}^{b}(x)=\left(\begin{array}{cccc}
\frac{1}{2 r_{s}} \sqrt{\frac{F(r)}{f(r)}} R & -\frac{c}{2 r_{s}} \sqrt{\frac{F(r)}{f(r)}} T & 0 & 0 \\
-\frac{c}{2 r_{s}} \sqrt{\frac{F(r)}{f(r)}} T & \frac{1}{2 r_{s}} \sqrt{\frac{F(r)}{f(r)}} R & 0 & 0 \\
0 & 0 & 1 & 0 \\
0 & 0 & 0 & 1
\end{array}\right) .
$$

Since this Lorentz transformation is a boost along the 1-axis parallel to the $r$-direction, the new local inertial frame falls into the black hole when $T>0$. To perform measurements in this local inertial frame, the observers also must fall into the black hole. Similarly, we choose the four-velocity of particles as

$$
\tilde{u}^{T}(x)=\frac{\cosh \tilde{\xi}}{\sqrt{F(r)}}, \quad \tilde{u}^{\phi}(x)= \pm \frac{c \sinh \tilde{\xi}}{r}
$$

which is not singular at the horizon. Since $\tilde{p}^{1}(x)=\tilde{e}_{\mu}^{1}(x) \tilde{u}^{\mu}(x)=0$, the particles also fall into the black hole with the local inertial frame (65), while moving in the $\pm \phi$-directions. With the vierbein (65) and four-velocity (67), we obtain the local Wigner rotation

$$
\begin{aligned}
\tilde{\vartheta}_{3}^{1}(x) & =-\tilde{\vartheta}^{3}{ }_{1}(x) \\
& = \pm \frac{c \cosh \tilde{\xi} \sinh \tilde{\xi}}{r}\left[3+\frac{r}{r_{s}}\right] \frac{\sqrt{F(r)} R}{4 r_{s}},
\end{aligned}
$$

instead of Eq. (44). Since this precession is not singular at the horizon, the observers on the horizon can extract the EPR correlation from the particles without the infinite accuracy of $\Phi$. Furthermore, they can extract the EPR correlation beyond the horizon $r<r_{s}$ (until the physical singularity $r=0$ ).

\subsection{Bell's Inequality}

We next consider Bell's inequality using the circularly moving particles (36) in the static local inertial frame (30). Suppose that the spin component of one particle is measured in the $(1,0,0)$-direction (component $\mathcal{Q})$ or in the $(0,1,0)$ direction (component $\mathcal{R}$ ) in the local inertial frame at $\phi=\Phi$, and suppose that the spin component of the other is measured in the $(-1,-1,0) / \sqrt{2}$ direction (component $\mathcal{S}$ ) or in the $(1,-1,0) / \sqrt{2}$-direction $($ component $\mathcal{T}$ ) in 
the local inertial frame at $\phi=-\Phi$. This set of observables gives rise to the maximal violation of Bell's inequality for the spin-singlet state in the usual case. However, for the circularly moving particles in the Schwarzschild spacetime, the degree of the violation of Bell's inequality apparently decreases as

$$
\langle\mathcal{Q S}\rangle+\langle\mathcal{R S}\rangle+\langle\mathcal{R} \mathcal{T}\rangle-\langle\mathcal{Q T}\rangle=2 \sqrt{2} \cos ^{2} \Theta
$$

Again, this result includes the effect of the trivial rotations of the local inertial frames $\pm \Phi$. To get rid of this effect, the observers rotate the directions of the measurement about the 2 -axis through the angles $\mp \Phi$, respectively. That is, the spin component of one particle is measured in the $(\cos \Phi, 0,-\sin \Phi)$ direction (component $\mathcal{Q}^{\prime}$ ) or in the $(0,1,0)$-direction (component $\mathcal{R}^{\prime}$ ), and the spin component of the other is measured in the $(-\cos \Phi,-1,-\sin \Phi) / \sqrt{2}-$ direction (component $\left.\mathcal{S}^{\prime}\right)$ or in the $(\cos \Phi,-1, \sin \Phi) / \sqrt{2}$-direction (component $\left.\mathcal{T}^{\prime}\right)$. Nevertheless, the degree of the violation of Bell's inequality still decreases as

$$
\left\langle\mathcal{Q}^{\prime} \mathcal{S}^{\prime}\right\rangle+\left\langle\mathcal{R}^{\prime} \mathcal{S}^{\prime}\right\rangle+\left\langle\mathcal{R}^{\prime} \mathcal{T}^{\prime}\right\rangle-\left\langle\mathcal{Q}^{\prime} \mathcal{T}^{\prime}\right\rangle=2 \sqrt{2} \cos ^{2} \Delta
$$

due to acceleration and gravity. Of course, local realistic theories cannot be restored, since Eq. (70) is a consequence of local unitary operations. This decrease means that it is a different set of directions that maximally violates Bell's inequality. To utilize the violation of Bell's inequality for quantum communication, the observers must take into account the general-relativistic effect arising from acceleration and gravity. More specifically, the spin component of one particle must be measured in the $(\cos \Theta, 0,-\sin \Theta)$-direction or in the $(0,1,0)$-direction in the local inertial frame at $\phi=\Phi$, and the spin component of the other must be measured in the $(-\cos \Theta,-1,-\sin \Theta) / \sqrt{2}$ direction or in the $(\cos \Theta,-1, \sin \Theta) / \sqrt{2}$-direction in the local inertial frame at $\phi=-\Phi$.

However, in practice, it becomes difficult to observe the violation of Bell's inequality when an uncertainty in $\Phi$ is near the horizon. Even if the directions of measurement are adjusted so that Bell's inequality is maximally violated, the error in $\Theta$ decreases the degree of violation as $2 \sqrt{2} \cos ^{2} \delta \Theta$. This value must be greater than 2 to verify the violation of Bell's inequality. Therefore, from Eq. (60), $\delta \Phi$ and $r$ must satisfy at least

$$
\delta \Phi<\sqrt{2}\left|1+\frac{\Delta}{\Phi}\right|^{-1}
$$


For a given uncertainty $\delta \Phi$, there exists a radius $r_{b}\left(>r_{s}\right)$ such that static observers at $r<r_{b}$ cannot observe the violation of Bell's inequality from circularly moving particles. To see the violation of Bell's inequality at $r<r_{b}$, the observers must fall into the black hole together with the particles, using the vierbein (65) and the four-velocity (67).

Using a different definition of a relativistic spin, Czachor [26] obtained a decrease in the degree of violation of Bell's inequality. This decrease was caused by the inertial motion of particles in Minkowski spacetime. In contrast to this result, Bell's inequality is unaffected except for a trivial rotation in our formulation in that case. Czachor's effect is thus different from ours. Terno [27] discussed a relation of different choices of relativistic spin operators to the violation of Bell's inequalities.

\section{Summary}

We considered the EPR correlation and the violation of Bell's inequality with accelerated particles in a gravitational field. Using relativistic quantum theory in curved spacetime, we explictly derived the local Wigner rotation during the motion of the particle. Considering particles in a circular motion in the Schwarzschild spacetime, we showed that acceleration and gravity deteriorate the EPR correlation in the directions that are the same in nonrelativistic theory, and apparently decrease the degree of the violation of Bell's inequality. This finding indicates neither a breakdown of the non-local correlation nor a restoration of local realistic theories. In fact, if the spins are measured in appropriately chosen different directions, we can obtain the perfect anti-correlation and the maximal violation of Bell's inequality. Our results mean that, in order to utilize the non-local correlation and the violation of Bell's inequality for quantum communication, we must take account of the relativistic effect by adjusting the directions of measurement; otherwise, the accuracy of quantum communication is reduced. In principle, we need information about the four-velocity and the vierbein in order for the communication to be perfect.

Moreover, we showed that near the event horizon even a small uncertainty in the identification of observers' positions results in a fatal error in identifying the measurement direction needed to maintain the perfect EPR correlation, because of an extremely rapid spin precession. In particular, static observers on the horizon can extract the EPR correlation from cir- 
cularly moving particles only if they have infinite accuracy as to their own positions. To exploit the EPR correlation on and beyond the horizon, the observers must choose a four-velocity and vierbein that are not singular at the horizon, and thus the observers must fall into the black hole together with the particles. This example demonstrates that the choices of four-velocity and vierbein are important to the ability to communicate non-locally in a curved spacetime using an EPR pair of spins.

\section{Acknowledgments}

H.T. was partially supported by JSPS Research Fellowships for Young Scientists. This research was supported by a Grant-in-Aid for Scientific Research (Grant No.15340129) by the Ministry of Education, Culture, Sports, Science and Technology of Japan, and by the Yamada Science Foundation.

\section{References}

[1] A. Einstein, B. Podolsky, and N. Rosen, Phys. Rev. 47, 777 (1935).

[2] D. Bohm, in Quantum Theory (Dover, New York, 1989), pp. 611-623.

[3] C. H. Bennett et al., Phys. Rev. Lett. 70, 1895 (1993).

[4] A. K. Ekert, Phys. Rev. Lett. 67, 661 (1991).

[5] C. H. Bennett, G. Brassard, and N. D. Mermin, Phys. Rev. Lett. 68, 557 (1992).

[6] A. Peres, P. F. Scudo, and D. R. Terno, Phys. Rev. Lett. 88, 230402 (2002).

[7] P. M. Alsing and G. J. Milburn, Quantum Inf. Comput. 2, 487 (2002).

[8] H. Terashima and M. Ueda, Quantum Inf. Comput. 3, 224 (2003); Int. J. Quantum Inform. 1, 93 (2003).

[9] J. Rembieliński and K. A. Smoliński, Phys. Rev. A 66, 052114 (2002).

[10] R. M. Gingrich and C. Adami, Phys. Rev. Lett. 89, 270402 (2002); R. M. Gingrich, A. J. Bergou, and C. Adami, Phys. Rev. A 68, 042102 (2003). 
[11] D. Ahn, H.-j. Lee, and S. W. Hwang, quant-ph/0207018 D. Ahn, H.-j. Lee, Y. H. Moon, and S. W. Hwang, Phys. Rev. A 67, 012103 (2003).

[12] H. Li and J. Du, Phys. Rev. A 68, 022108 (2003).

[13] E. P. Wigner, Ann. Math. 40, 149 (1939).

[14] J. S. Bell, Physics 1, 195 (1964).

[15] J. F. Clauser, M. A. Horne, A. Shimony, and R. A. Holt, Phys. Rev. Lett. 23, 880 (1969).

[16] H. von Borzeszkowski and M. B. Mensky, Phys. Lett. A 269, 197 (2000).

[17] S. J. van Enk and T. Rudolph, Quantum Inf. Comput. 3, 423 (2003).

[18] P. M. Alsing and G. J. Milburn, Phys. Rev. Lett. 91, 180404 (2003).

[19] S. Takagi, Prog. Theor. Phys. Suppl. 88, 1 (1986).

[20] M. Nakahara, Geometry, Topology and Physics (Institute of Physics Publishing, Bristol, 1990).

[21] N. D. Birrell and P. C. W. Davies, Quantum Fields in Curved Space (Cambridge University Press, Cambridge, 1982).

[22] S. W. Hawking, Commun. Math. Phys. 43, 199 (1975).

[23] S. Weinberg, The Quantum Theory of Fields (Cambridge University Press, Cambridge, 1995).

[24] Y. Ohnuki, Unitary Representations of the Poincaré Group and Relativistic Wave Equations (World Scientific, Singapore, 1988).

[25] R. M. Wald, General Relativity (University of Chicago Press, Chicago, 1984).

[26] M. Czachor, Phys. Rev. A 55, 72 (1997).

[27] D. R. Terno, Phys. Rev. A 67, 014102 (2003). 